\title{
Honey, I shrunk the lab
}

\author{
Take a lab full of test tubes, flasks and \\ stirrers, and cram it onto a silicon chip - \\ that's the dream of microfluidics \\ researchers. And as Jonathan
} Knight finds out, this vision is becoming a practical reality.

A bout a decade ago, a handful of researchers began discussing an intriguing idea. Could the equipment needed for everyday chemistry and biology procedures be shrunk to fit on a chip the size of a postage stamp? Miniature devices for, say, analysing proteins should be faster and cheaper than conventional versions. Government funding agencies, the military and venture capitalists signed up to the vision, and millions of dollars poured into 'lab-on-a-chip' projects.

But making miniature labs is not just a question of scaling down conventional equipment. Nanolitre volumes of liquid behave in odd ways. For example, we are used to seeing liquids mix by turbulent flow - as illustrated by the way cream swirls into coffee (see right). But such turbulence does not occur in channels just a few tens of micrometres wide. Streams of coffee and cream flow alongside each other over short distances without mixing, almost as if they were separated by glass.

Microfluidics, as the field that deals with this challenge is called, has in recent years made impressive progress in tackling problems such as mixing. Some of the stumbling blocks have been overcome, and basic chips are now on the market. Users confirm that routine tasks can be carried out faster than previously possible. Other problems with miniaturization remain the focus of an intense research effort, which now has its own journal — Lab on a Chip, published by Britain's Royal Society of Chemistry.

By definition, microfluidic systems save on reagents. But speed is a more important advantage. The sorting of protein or DNA samples into different sizes by gel electrophoresis, in which an electric field is used to pull the molecules through a gel, takes at least half an hour on the bench but no more than 30 seconds on a chip, for example. And when completely filled with fluid, microfluidic chambers and channels measure volumes more consistently than human hands can, thus reducing error rates.

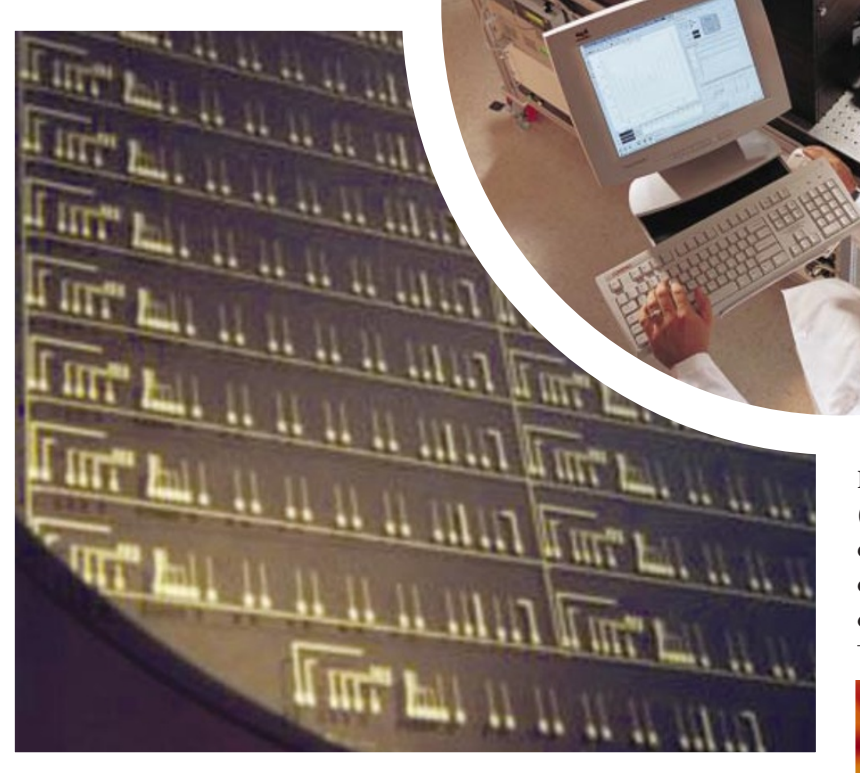

Fitting a lab onto a chip (top and left) often relies on getting tiny volumes of liquids to mix the way coffee and cream do.

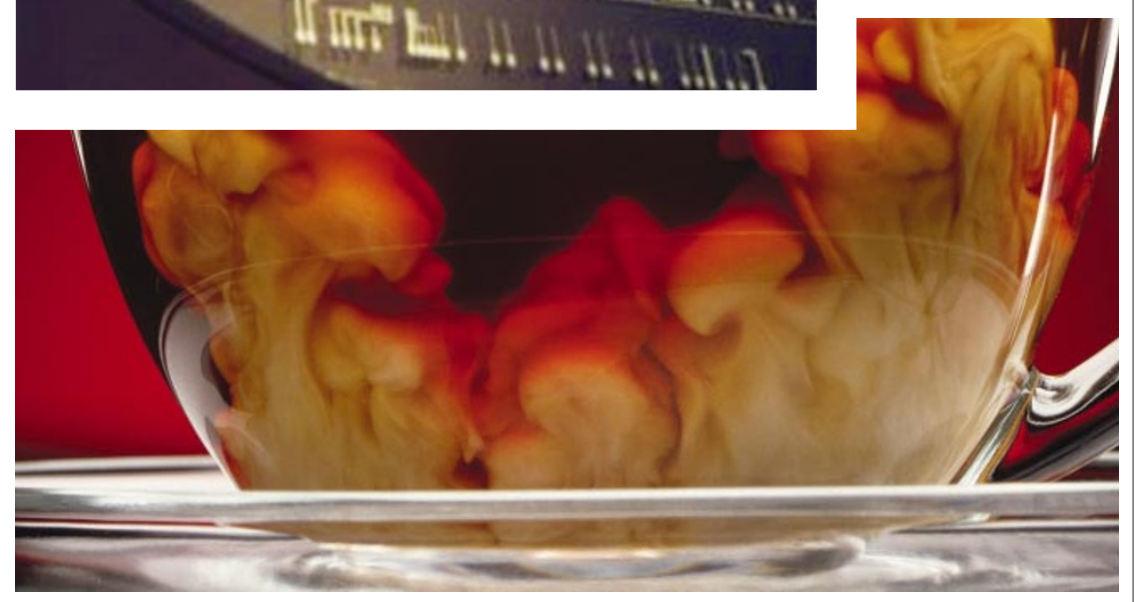

Building the chips is straightforward. The same etching techniques used to make semiconductor chips can create channels and chambers in silicon or glass wafers, which can be layered on top of one other to make more complex patterns. Chip designs can also be stamped or cut into plastic sheets. With the help of computer-design software, researchers can go from concept to prototype in days or hours.

\section{A tight squeeze}

But having made a chip, convincing liquid samples to move around the tiny channels is not as easy as it would seem. Air pressure can push samples through channels, but if the sample consists of a concentrated dollop of protein, say, this becomes a problem.
The channel walls exert a drag on the liquid, so that fluid at the centre of the channel moves faster than that at the edge and concentrated samples quickly become smeared.

The most commonly used alternative makes use of a phenomenon called electroosmosis. In the case of microfluidics, this effect occurs because the channel wall ionizes water molecules near it by donating protons to them. If an electric field is applied along the channel, these positively charged ions flow towards the negative pole and drag the rest of the fluid along with them. Because the pull comes from the edge, the fluid moves as one. Electro-osmosis is widely used in experimental microfluidic chips, and might even turn up in home computers, as Stanford University engineers Daniel Laser and Juan 
Santiago are developing a liquid-cooling system based on electro-osmosis to transfer heat away from microprocessors.

The mechanism behind electrophoresis - the pull exerted by an electric field on charged proteins or DNA — can also be used to move samples around chips. Caliper Technologies, a microfluidics company based in Mountain View, California, makes a gel-electrophoresis chip. Samples are pulled through channels containing a liquid polymer, which sifts biological molecules such as proteins just as conventional gels do. The polymer inhibits electro-osmosis - possibly by clinging to the channel walls - but the pull of the electric field on the sample is enough to load the chip, saturating a 100-micrometre-wide channel with DNA or protein.

\section{Split screen}

Once full, the direction of the electric field is shifted so that some of sample is pulled into a side channel where separation can occur. Bands of the DNA or protein, which were stained beforehand with a fluorescent dye, are identified by illuminating the channel with a laser. A single sample can be processed in about 20 seconds.

Persuading liquids in the chips to mix remains one of the biggest problems facing microfluidics researchers. Liquids mix by diffusing into each other. For large volumes, this process can be sped up by stirring, because the turbulence caused increases the area of the interface between the liquids.

But turbulent flow faces opposition in the shape of the viscosity of the two liquids, which tends to keep fluid motion stable. If the sample is sufficiently small, it will never generate the momentum needed to overcome this obstacle. At the microfluidic level, mixing liquids is like trying to stir molasses into honey, and two liquids travelling side-byside through a narrow channel only become fully mixed after many centimetres.

One approach to accelerating the process is to stretch and fold the fluid layers as they move down the channel, like kneading a ball of dough. In January, George Whitesides' group at Harvard University achieved this using a herringbone pattern of ridges on the channel's floor ${ }^{1}$. The ridges rotate the flow clockwise on the left side and anticlockwise on the right, causing fluid at the centre to fold in on itself. The mix is complete in only $3 \mathrm{~cm}$ — short enough to fit on a chip if a winding channel is used. Without the ridges, the liquids would only have mixed after $100 \mathrm{~cm}$.

Michael Oddy, a microfluidics engineer in Santiago's Stanford lab, discovered another mixing technique while experimenting with electro-osmosis. He found that applying an alternating current causes the fluid to oscillate in the channel, and that the oscillation is unstable at certain frequencies ${ }^{2}$. The details of why this happens remain unclear, but mixing times are much reduced. "We get a nearly homogeneous mixture in about 3 seconds, compared with 500 seconds for diffusion alone," says Santiago.

Other researchers are finding that the lack of mixing can sometimes be an advantage. Paul Yager, a bioengineer at the University of Washington in Seattle, has developed a device for measuring the concentration of solutions of small molecules such as peptides $^{3}$. In his chip, two solutions flow into the two arms at the top of a T-shaped channel 1,200 micrometres wide. One solution contains the molecule of interest and the other a larger antibody that binds to it.

\section{Separate streams}

When the liquids meet and head down the main channel, the lack of turbulence ensures that mixing occurs only by diffusion. But the few smaller molecules that diffuse into the neighbouring layer bind to the much larger antibody molecules, which effectively halt the movements of the molecules they capture. The higher the concentration of small molecules, the more of them escape capture and are able to diffuse deeper into the antibody solution. By tagging a few of the small molecules with a fluorescent label, the diffusion rate, and hence the concentration, can be revealed by illuminating the antibody solution with a laser.

But making a true lab on a chip means combining several processes. At the University of Michigan, chemical engineer Mark Burns has produced an electrophoresis chip that does just that ${ }^{4}$. Two parallel channels hold precise volumes of liquid, such as a stained solution of DNA and an enzyme that can cleave DNA. Air pressure is used to push the samples into a chamber, where a microelectronic heater warms them to the optimal reaction temperature. They are incubated for about 15 minutes, and then loaded into a channel containing a gel for electrophoresis.

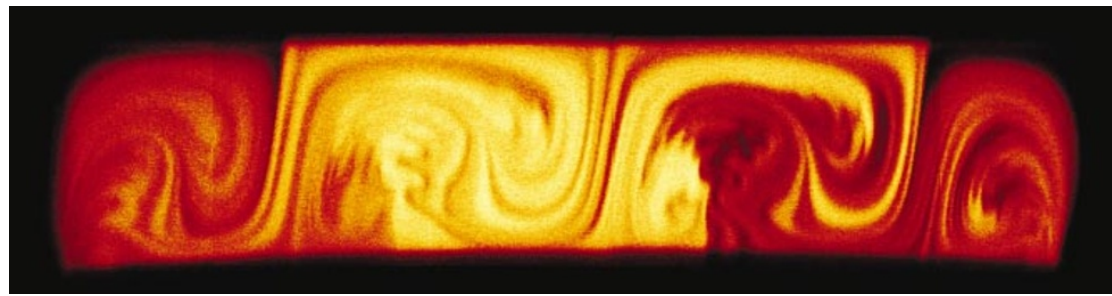

Meeting a knead: small ridges in microchannels can force liquids to mix by folding them together.

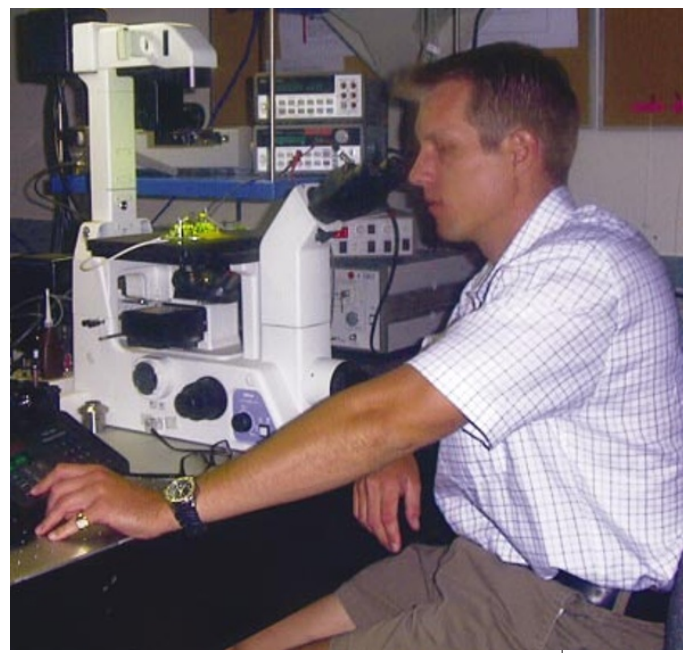

A whole lotta shakin': Michael Oddy uses an alternating current to mix liquids by oscillation.

Finally, a photodetector notes fluorescent bands of DNA fragments in the gel corresponding to specific DNA sequences that were present in the original sample.

For some users, there is no need to cram all of this hardware onto the chip. The Caliper chips, for instance, use a toastersized instrument to house the laser and the electronics to run the electrophoresis. Pharmaceutical researchers, for example, don't care if the equipment fills the room, as long as it is fast and accurate, says Andreas Manz, a chemist at Imperial College London, and one of the pioneers of microfluidics.

But others see portability as an essential part of the future of microfluidics. Yager, whose concentration sensor is being commercialized by Micronics, a Redmondbased company that was spun off from the University of Washington in 1996, sees a huge market in home healthcare. He suggests that his device could be used to conduct blood tests with only a pinprick sample, for example.

Burns, meanwhile, believes that his chips could ultimately be developed into a home kit to test for bacterial DNA. Users could plug the chips into a laptop, and e-mail the results to their doctor. "We're building the expertise into the chip," says Burns. If such predictions turn out to be correct, the new chips won't just be shrunken labs - they will also embody the skills of laboratory technicians.

Jonathan Knight writes for Nature from San Francisco.

1. Stroock, A. D. et al. Science 295, 647-651 (2002).

2. Oddy, M. H., Santiago, J. G. \& Mikkelsen, J. C. Anal. Chem. 73, 5822-5832 (2001).

3. Hatch, A. et al. Nature Biotechnol. 19, 461-465 (2001)

4. Burns, M. A. et al. Science 282, 484-487 (1998).

\section{Lab on a Chip}

www.rsc.org/is/journals/current/loc/locpub.htm Caliper Technologies

www.calipertech.com

Micronics

www.micronics.net 